\title{
Comment on "Signatures of the Unruh effect via high-power, short-pulse lasers"
}

by P.G. Thirolf, D. Habs, A. Henig, D. Jung, D. Kiefer, C. Lang, C. Schaller, J. Schreiber, and R. Schuetzhold Eur. Phys. J. D 55, 377-387 (2009)

The editors are aware of the ongoing discussion on the role of the Unruh and Hawking effects in the two-photon emission process of an electron in a strong electromagnetic field. The current article discusses the possibilities for experimental detection of the Unruh effect in the laboratory and its close relation with the development of ultra-high intensity laser plasma interaction. For this important reason the editors decided to publish it within the topical issue "Fundamental physics and ultra-high laser fields". The additional aim of this publication is to encourage discussion of this problem on the pages of our journal. 